\title{
Mixed Valence Model for Superconductivity
}

\author{
Sven Larsson \\ Department of Chemistry, Chalmers University of Technology \\ SE-41296 Göteborg, Sweden
}

Received on 23 May, 2003.

\begin{abstract}
Superconductivity often occurs in crystals with one active electron per site with charge density wave (CDW) or spin density wave (SDW) as 'mother state'. It is proposed that superconductivity is possible when the differences in equilibrium geometry and energy between the diabatic CDW and SDW states are so small that there is interaction between them via the zero point vibrations. Electron pairing in real space is directly related to oxidation states being different in two units. Three valence states in succession have to be stable (ground state or low-energy excited states) and we therefore refer to this mixed valence model as the MV-3 model. Examples are chosen from bismuthates, cuprates, and fullerides. The theory is simple and straightforward and offers solutions to other important problems as well, for example for $A_{3} C_{60}(A=K, R b)$, that (1) there are no magnetic moments in crystal phase, and (2) that these systems are superconducting metals while $A_{4} C_{60}$ are insulators.
\end{abstract}

\section{Introduction}

Since small coherence length characterizes 'exotic' superconductivity, it is possible to start out a theoretical description from local electronic structure and electron-nuclear interactions, and later extend the system and wave function to the infinite crystal. If we do so we obtain a highly 'chemical' description of moving electron pairs $[1,2]$. The concept of oxidation state is relevant here; in particular the existence of oxidation states of atoms or molecules that are different in two units, since this obviously has to do with electron pairing. Crystalline systems with on the average one electron per site, but with alternating valency, are called charge density wave (CDW), 'negative-U', or mixed valence (MV) states. The MV states of relevance here may be obtained in the following disproportionation reaction:

$$
2 M^{n+} \leftrightarrow M^{(n+1)+}+M^{(n-1)+}
$$

On the left side there is one electron per site. If antiferromagnetic this state is referred to as a spin density wave (SDW) state. Successful theories for mixed valence systems have existed for a long time [4] and have been connected to [5] theories for electron transfer (ET) [6] and transport [7] and superconductivity [2,3,8-11], even before the advent of high $T_{c}$ superconductors [8]. This theory allows a quantum mechanical treatment of nuclear motion, including the coupling between nuclear and electronic motion (electronphonon interaction) [12-13]. ET mixes two successive valence states while electron pair transfer (EPT) mixes two valence states different in two units. A non-trivial theorem states that the free energy of reaction (1) has to be close to zero $\left(\Delta G_{0} \sim 0\right)$ to permit electron pairs to be transported without activation energy [3]. Hence three valence states in succession have to be involved. The two MV cases will be called MV-2 and MV-3 systems, respectively. If in addition to the condition $\Delta G_{0} \sim 0$ the equilibrium geometries of the CDW and SDW states are similar, new vibronic states are formed with components from both CDW and SDW diabatic states. In this limit the electron pairs are delocalised. The free motion of electron pairs is without resistance since there is an energy gap to the first excited state. Furthermore the difference in oxidation state is no longer observable. Unfortunately the latter fact has lead to some confusion on the connection between superconducting and MV systems. A theory for electronic localization has been set up [14-16] on the basis of coupling between electronic and nuclear motion. In this theory reorganization energy $\lambda$ 'competes' with coupling energy $\Delta . \Delta=2 t$, where $\mathrm{t}$ is the coupling. $\lambda$ is unrelated to $\mathrm{U}$ of Mott theory. Localization cannot be due to purely electronic effects, although it may look like that by the way the wave function is expressed mathematically, or by the fact that the wave function is not sufficiently accurate. A well-known example is K-shell ionisation in a homonuclear diatomic molecule. The unrestricted Hartree-Fock method leads to symmetry breaking with a localized hole [17]. However, the symmetrized wave function is obviously more accurate and has a symmetric (delocalised) 1s-hole. The hole cannot be localized to a single atom in a homonuclear diatomic molecule since symmetry is conserved under the only degree of freedom (bond length). Concerning $\mathrm{U}$, it is an experimental parameter $(\mathrm{U}=\mathrm{I}-\mathrm{A})$ without predictive capability. A theory based on $\lambda$ and $\Delta$, on the other hand, can predict localization in for example:

1. $\left[\left(\mathrm{NH}_{3}\right)_{5} \mathrm{Ru}-\text { pyrazine }-\mathrm{Ru}\left(\mathrm{NH}_{3}\right)_{5}\right]^{5+}$

2. $\left[\left(\mathrm{NH}_{3}\right)_{5} \mathrm{Ru}-\text { dipyridine }-\mathrm{Ru}\left(\mathrm{NH}_{3}\right)_{5}\right]^{5+}$ 
Localization by modification of the RuN bond lengths has equal chance in the two complexes. Creutz showed experimentally that system 1 . with a pyrazine bridge is delocalised [18] with equal $\mathrm{Ru}$ valencies $\left(R u^{2.5+}-R u^{2.5+}\right)$, since $\lambda<\Delta$. In system 2 . with the longer dipyridine bridge, $\lambda>>\Delta$, and the valencies localized $\left(R u^{2+}-R u^{3+}\right)$. A quantum chemical calculation of wave function and geometry gives the same result, of course. The mentioned delocalisation concept may be extended to infinite systems [16]. Only two parameters are needed to decide whether the system is localized or delocalised: reorganization energy $(\lambda)$ and effective coupling $(\Delta / 2) . \lambda$ and $\Delta$ may be calculated in the finite dimer and monomer systems, respectively. Below we will sketch MV-3 theory as applied to superconductivity. Subsequently examples will be given from three large groups of exotic superconductors: bismuthates, cuprates, and fullerides. We will emphasize that the same theory can be used for these rather different examples taken from inor- ganic as well as organic chemistry.

\section{Theory}

Atoms or molecules in different oxidation states are completely different chemical species that are stable in different environments. In the case of superconductivity we are concerned with transfer of two electrons from one site to another, in a delocalised fashion. The vibrational motion connected to this distortion is illustrated in Fig. 1. If the distortion of the nuclear sites is large there will be an activation barrier, destructive for superconductivity. A small distortion on the other hand leads to a coupling between electron and nuclear motion, promoting superconductivity. Potential energy surfaces are seen in Figs. 2 and 3. The reorganization energy $\lambda$ is a measure of the distortion which may be calculated as follows:

$$
\lambda=\sum_{i} \frac{1}{2} k_{i} \delta d_{i}^{2} \approx\left\{\begin{array}{rrrr}
15 \sum_{M L b o n d s} \delta d_{i}^{2} & (\mathrm{eV}) & \text { (metal } & \text { complexes }) \\
24 \sum_{C C b o n d s} \delta d_{i}^{2} & (\mathrm{eV}) & \text { (organic } & \pi-\text { systems })
\end{array}\right.
$$

$\delta d_{i}(\AA)$ is the change in the i'th bond length. The summation extends over all bonds in donor and acceptor, but the important ones will be metal-ligand (ML) bonds in metal complexes and carbon-carbon (CC) bonds in organic $\pi$ systems. Approximate values are given in the two cases, where 'normal' bond strength applies. For example if two bonds change by $0.1 \AA$ in a metal complex, the reorganization energy $\lambda \sim 0.3 \mathrm{eV}$. In other cases described below the changes may be considerably larger, say $0.2 \AA$ in twelve bonds, six on each site. We then obtain $\lambda \sim 7.2 \mathrm{eV}$. Since the activation energy is $\lambda / 4$ [6] we obtain barriers of $<0.1$ and $1.8 \mathrm{eV}$ in the two cases, leading to very different behaviour. In the case of organic $\pi$-systems we usually obtain $\lambda<0.4 \mathrm{eV}$. When interactions between the sites are 'switched on' the potential energy surface (PES) intersections become avoided crossings (Figs.2,3). In the MV-2 case where the valence state changes by one unit [6], the activation barrier $E_{a}$ decreases from $\lambda / 4$ if $|\Delta|$ is increased from zero, and vanishes for $\lambda=|\Delta|$ :

$$
E_{a}=\frac{(\lambda-|\Delta|)^{2}}{4 \lambda}, \quad \text { if } \quad|\Delta|<\lambda
$$

In the MV-3 case the situation is more complicated. The coupling between the two-electron PES's is very small and this necessitates the SDW PES to intersect (Fig.3) the CDW diabatic states [3]. When the barrier vanishes the valencies are delocalized and the character of the visible spectrum tends to change to black. It should be noted that mixed valence theory is completely consistent with the ordinary quantum mechanical approach used in chemistry ('quantum chemistry'). Bond length changes and reorganization en- ergy may be calculated in principle. The PES given in Figs. 1 and 2 are Born-Oppenheimer PES. There are no pairing forces other than those normally included in the electronic Hamiltonian. The connection to the BCS model [19] may be realized if a $\mathrm{k}$-space expansion is performed.

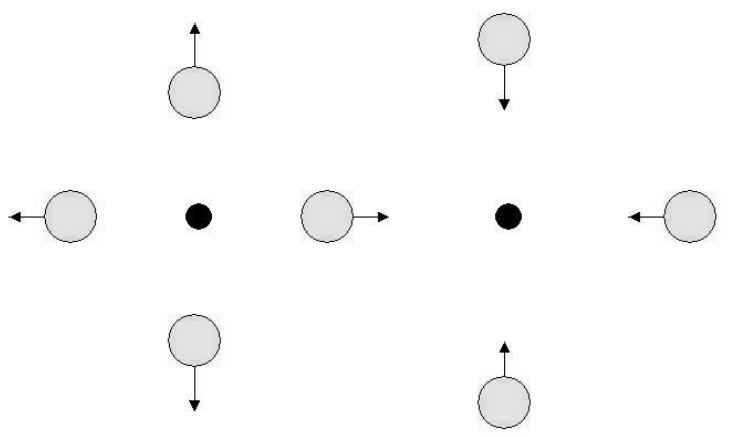

Figure 1. Active vibrational modes for electron pair transfer.

\section{Electron pairing in real space}

It is important to know in what type of chemical structure electron pairs can be accommodated and move without great structural changes. There is general agreement that in transition metal ions the $\mathrm{d}$ electrons and in aromatic molecules the $\pi$ electrons are 'active', and that the number of $\mathrm{d}$ or $\pi$ 


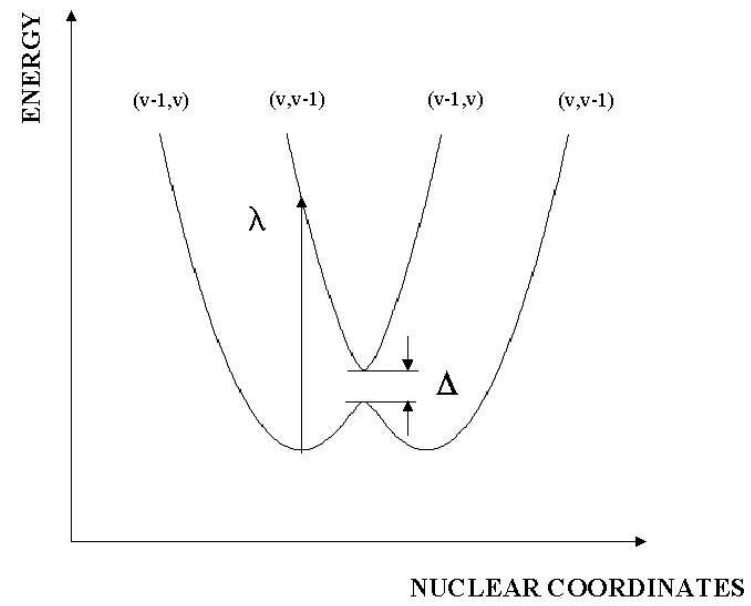

Figure 2. Potential energy curves in the MV-2 case. $\lambda$ is the reorganization energy. The left parabola corresponds to the oxidation states $\mathrm{v}$ and $\mathrm{v}+1$ on the two sites and the right parabola to oxidation states $\mathrm{v}+1$ and $\mathrm{v}$.

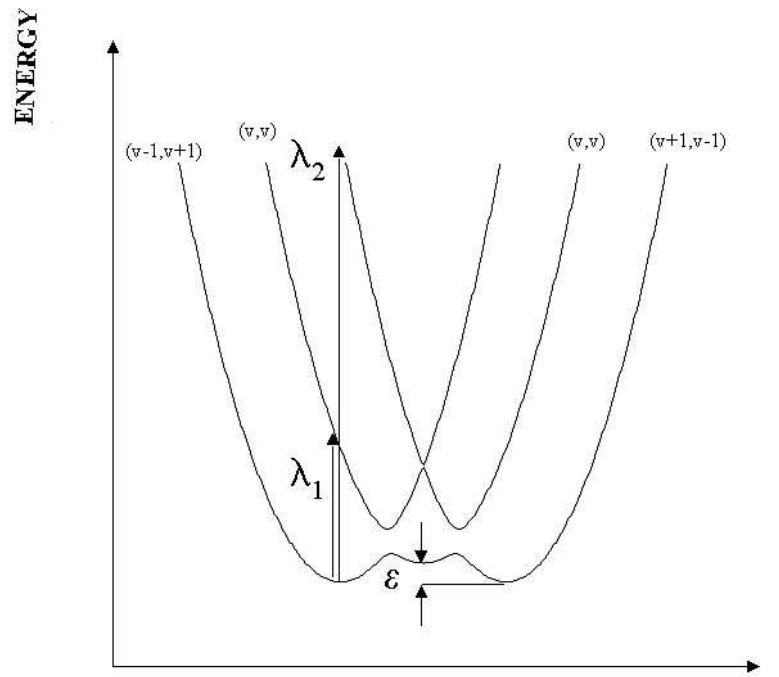

NUCLEAR COORDINATES

Figure 3. Potential energy curves in the MV-3 case. $\lambda_{1}$ and $\lambda_{2}$ are reorganization energies for transfer of one or two electrons, respectively. $\mathrm{v}$ is the valence state of the comproportionated state and $\mathrm{v}-1$ and $\mathrm{v}+1$ of the disproportionated state. The latter state is assumed to be lower in energy in the figure (negative-U state).

electrons can change without a great change of structure. Also $n s^{2}$ or $n p^{2}$ pairs of electrons have been implicated. In planar $M g B_{2}$ both $\sigma$ and $\pi$ electrons are involved [20]. Below we will choose examples from three major groups of exotic superconductors and find that the same MV-3 theory may be applied.

\section{1 $6 s^{2}$ electron pairs in bismuthates}

A typical example is $\mathrm{BaBiO}_{3}$. This system becomes superconducting if doped, as $\mathrm{BaPb}_{x} \mathrm{Bi}_{1-x} \mathrm{O}_{3}(x \sim 0.3)$ [21] and $\mathrm{Ba}_{1-x} \mathrm{~K}_{x} \mathrm{BiO}_{3}(x \sim 0.4)$ [22]. The $\mathrm{Bi}$ sites in $\mathrm{BaBiO}_{3}$ are alternating $\mathrm{Bi}^{3+}$ and $B i^{5+}$, i e with the sites occupied by a $6 s^{2}$ pair or unoccupied, respectively. Since the $6 \mathrm{~s}$ orbital has a quite large extension the $\mathrm{BiO}$ distance has to be larger in the $B i^{3+}$ case than in the $B i^{5+}$ case. The enlargement region is roughly where the $6 \mathrm{~s}$ orbital is localized. This CDW system needs to interact with a SDW state with single electron occupancy, i e $\mathrm{Bi}^{4+}$ sites [3]. Doping leads to decreased differences in bond length [23] and this makes superconductivity possible [3]. There is general agreement that superconductivity in $\mathrm{BaPb}_{x} \mathrm{Bi}_{1-x} \mathrm{O}_{3}$ and $\mathrm{Ba}_{1-x} \mathrm{~K}_{x} \mathrm{BiO}_{3}$ can be explained as indicated above [2,3,911]. The absence of superconductivity in the CDW case $\mathrm{Cs}_{2} \mathrm{SbCl}_{6}$ [24] is also easily explained as due to too large activation energy, which in its turn depends on great difference in bond lengths between the $S b^{3+}$ and $S b^{5+}$ sites.

\section{2 $3 d^{2}$ hole pairs in cuprates}

$\mathrm{Cu}, \mathrm{Ag}$, and $\mathrm{Au}$ are often stable in two or three oxidation states. In square planar geometry the ground states for $C u^{2+}$ and $\mathrm{Cu}^{3+}$ correspond to one or two missing electrons, respectively, in the antibonding molecular orbital (MO) with $3 d\left(x^{2}-y^{2}\right)$ character. The Jahn-Teller theorem implies smaller $\mathrm{CuO}$ distances in the xy-plane with slightly longer bond length for $C u^{2+}$ than for $C u^{3+}$. Consistency with theory would require that $C u^{+}$can exist at a near octahedral geometry with not too different bond lengths, making the CDW state reasonably stable. The one electron per site case, corresponding to $C u^{2+}$, is antiferromagnetic in $\mathrm{La}_{2-x} \mathrm{Ba}_{x} \mathrm{CuO}_{4}(x=0)$ and $\mathrm{YBa}_{2} \mathrm{Cu}_{3} \mathrm{O}_{7-x}(x=0.5)$, but for somewhat larger average oxidation state than +2 both systems become superconducting $[25,26]$. This suggests, in the same way as for bismuthates, that the energy difference between the SDW and CDW states depends on doping and (hypothetically) tends to zero in certain doping ranges. In most $C u^{2+}$ systems the SDW state (or paramagnetic spin states) are more stable than the disproportionated state for $\mathrm{Cu}^{2+}$ oxides and halides. In the cuprates it seems reasonable that the $\mathrm{CuO}_{2}$ layer has its oxidation level fine tuned from the intervening layer, which thus regulates also the relative energies of the CDW and SDW states. In the case of silver and gold, the disproportionated mixed valence state tends to be more stable. Still, however, $A g F_{2}$ has a stable phase at low temperature called $\alpha-A g F_{2}$. It has a typical Jahn-Teller distortion with four short bonds (2.07) and two long bonds (2.58). Contrary to the case in $C u F_{2}$ the structure is a puckered sheet. The spin structure is complicated [27]. $\beta-A g F_{2}$ is stable at a higher temperature and has mixed valence with a CDW ground state [28]. The two phases have very different structures. There is a phase transition at elevated temperatures. Since the energies of the SDW and CDW states are not the same at low temperature and the geometries very different, superconductivity cannot occur. In the layered compounds $C s A u X_{3}$ or $C s_{2} A u X_{4}$ $(\mathrm{X}=\mathrm{Cl}, \mathrm{Br}, \mathrm{I})$, the geometry suggests an $\mathrm{Au}(\mathrm{I})-\mathrm{Au}(\mathrm{III})$ mixed valence state [29]. The electron count corresponds to exactly one hole per site. $C s A u X_{3}$ or $C s_{2} A u X_{4}$ are 
semiconductors (at low pressure) with large activation energy [30,31]. The conductivity is increased by several orders of magnitude at high pressures of a few GPa [30-33], but superconductivity has not been reported. High pressure leads to decreased activation barrier (see below) and at the same time to an insulator to metal transition, of obvious relevance also to superconductivity [3,16]. Well's salt, $C s A u C l_{3}$, is probably the first and best-known example of disproportionation in gold compounds [29-33]. At normal pressure the distances of the xy-plane are 0.4 smaller in $\mathrm{Au}(\mathrm{III})-\mathrm{Cl}$ than corresponding distances for $\mathrm{Au}(\mathrm{I})-\mathrm{Cl}$. Along the $\mathrm{z}$-axis only $\mathrm{Au}(\mathrm{I})$ forms a bond to $\mathrm{Cl}^{-}$. The distances are 2.31 for $\mathrm{Au}(\mathrm{I})-\mathrm{Cl}$ and 3.13 for $\mathrm{Au}(\mathrm{III})-\mathrm{Cl}$ [29]. In $\mathrm{CsAuCl}$ the activation barrier for direct electron pair transfer (EPT) is hence very large. Pressure forces the geometric differences between different oxidation states to become smaller which means that the parabolas in Fig. 3 come closer to each other and hence that the activation barrier becomes smaller. The increase of $\Delta$ as the distance between the metal atoms is decreased is of less importance in this ase. Since $A A u X_{3}$ and $A_{2} A u X_{4}$ (A=alkali atom) compounds are iso-structural with $\mathrm{BaBiO}_{3}$ [33], superconductivity might be expected. An important difference to the bismuthates is the larger distance differences and that there does not seem to be any doped Au compound, which would provide a greater possibility for superconductivity. The doping could be with $P t^{2+}$ ions, which are also $d^{8}$ systems with low spin, but this has probably never been tried. Since Jahn-Teller effects act differently on different oxidation states, only systems where they 'cooperate' can be superconducting. In the planar cuprate case the hole state is always in the $\mathrm{CuO} 2_{2}^{-}$ plane for $\mathrm{Cu}^{2+}$ and $\mathrm{Cu}^{3+}$. Essentially only four bonds are changed and no bond angles, and hence $\lambda$ is quite small.

\section{3 $t_{1 u}^{2}$ electron pairs in fullerides}

In fullerenes the interactions between the atoms of the same site (disregarding the alkali ions) are covalent while the interactions between the sites are very small. The band-width, corresponding to interaction between the lowest unoccupied molecular orbital (LUMO) on different sites is in the range $0.1-0.2 \mathrm{eV}$ [34]. Hence a localized model should be particularly favourable in this case as a zero-order approximation. The fullerenes themselves usually Jahn-Teller distort to a non-degenerate ground state and hence they are not superconducting, in accordance with the model [3]. There is general agreement that alkali atoms donate their electrons to the fullerene molecules in, for example, superconducting $K_{3} C_{60}\left(T_{c} \sim 30 K\right)$ [35] and insulating $K_{4} C_{60}$ [36]. In $C_{60}$ the donated electrons enter a three-fold degenerate $\mathrm{MO}$ of symmetry $t_{1 u}$. This MO is thus half filled in $K_{3} C_{60}$ and 2/3 filled in $K_{4} C_{60}$. According to the elementary Hund's rule, high spin would be expected to prevail, but it does not. Both $K_{3} C_{60}$ and $K_{4} C_{60}$ are without magnetic moment at a low temperature [37]. Since the $C_{60}^{3-}$ molecule ion by itself in any case must have a magnetic moment, $K_{3} C_{60}$ would be expected to be ferro- or antiferro-magnetic, but is a metal, superconducting below about $\sim 30 \mathrm{~K}$. Band theory, of course, predicts metals in both cases, since the Fermi level is in the valence band. To explain that $K_{4} C_{60}$ is not a metal one has resorted to the Mott theory for metal - insulator transitions [38]. Han and Gunnarsson point out that the Mott electron repulsion parameter $\mathrm{U}$ is about the same in $K_{3} C_{60}$ and $K_{4} C_{60}$ [37], so why is not $K_{3} C_{60}$ an insulator? They argued that Jahn-Teller effects are stronger in low-spin than in high spin, concluding that low spin should be favoured in both cases. They give no particularly clearcut explanation for the metal - insulator problem [37]. A rather extensive study of reorganization energy and JahnTeller effect has been carried out for the fullerenes [39]. It was found that the Jahn-Teller effects are usually small and supporting low spin. This agrees with the conclusion in ref.[37] although it appears less likely that these small corrections can explain the large difference in localization properties between $K_{3} C_{60}$ and $K_{4} C_{60}$. It is also probable that the Jahn-Teller effects are too small to explain even the low spin problem. In fact Han and Gunnarsson [37] have neglected electron correlation which in this case completely off-sets Hund's rule [40]. Correlation effects are thus the most important reason why low spin prevails.

It still remains to explain why the spin of $K_{3} C_{60}$ is exactly zero (at a low temperature) and why it is a superconducting metal while $K_{4} C_{60}$ is an insulator. This explanation is simple and was suggested more than ten years ago [40], but apparently this has passed unnoticed. (In fact MV theories seem to be virtually unknown in the physics community!) In the case of $K_{3} C_{60}$ the disproportionation reaction is:

$$
2 C_{60}^{3-} \rightarrow C_{60}^{2-}+C_{60}^{4-}
$$

$C_{60}^{2-}$ and $C_{60}^{4-}$ should be low spin for the reasons stated above [40], also consistent with experimental data [41]. This makes it very likely that $C_{60}^{2-}$ and $C_{60}^{4-}$ also exist (probably delocalised) in $K_{3} C_{60}$ and that disproportionation is the explanation why $K_{3} C_{60}$ is without magnetic moment and superconducting. In this case the geometric differences between $C_{60}^{2-}, C_{60}^{3-}$, and $C_{60}^{4-}$ are small and lead to small reorganization energies of less than $0.1 \mathrm{eV}$ [39]. The coupling for this reason can be allowed to be quite small to achieve a delocalising interaction between CDW, SDW and vibrational zero point motions. It is this interaction that implies that the whole system is without moments. Accurate calculations can be done in principle to calculate coupling and reorganization energy. It should be mentioned that also the alkali ions are likely to leave a contribution to the reorganization energy. This part can also be calculated, although it is difficult. It is certain that the positional variation, depending on the charges of the surrounding $C_{60}$ molecules, is greater for the smaller alkali ions. Hence the contribution to the reorganization energy is much larger for the smaller alkali ions. This may partly explain why $N a_{3} C_{60}$ is not superconducting $[41,42]$. This explanation is supported by the fact that $N a_{3} C_{60}$ appears to be phase separated into $N a_{2} C_{60}$ and $N a_{6} C_{60}$ [41]. However, there are many other important facts to take into account in a discussion of alkali 
fullerides and superconductivity. We refer to a recent paper by Margadonna and Prassides [43]. If $\lambda$ is quite large compared to $\Delta$, then, at a high temperature the electrons would not be delocalised by the ordinary mechanism. Here again accurate calculations are necessary. Probably $K_{3} C_{60}$ is delocalised on account of the disproportionation mechanism eq.(2), while $K_{4} C_{60}$ (and $N a_{2} C_{60}[42,44]$ ) remains a semiconductor. The latter mechanism also explains why $K_{3} C_{60}$ is a superconductor. To use Mott theory to explain that $A_{3} C_{60}$ is a metal is doubtful in the mind of the present author, and superconductivity does not follow as a corollary of course. Why are other fullerides not superconducting? The answer is probably that the doping has to be done in such a way that the cubic (or possibly tetragonal) structure is maintained. At the same time the number of added electrons in LUMO (or possibly subtracted in HOMO) should allow a degeneracy to remain to permit formation of CDW and SDW states. These are severe conditions but there seem to be no compelling reasons why other doped fullerenes than those containing $K_{3} C_{60}$ cannot be superconductors.

\section{Conclusions}

We have discussed a theory for superconductivity [2,3] on local concepts used in chemistry, emanating from mixed valence theory [4-11]. As a result we can rather well understand why superconductivity appears in some systems but not in others. Since the theoretical treatment starts from a local treatment, it has been possible to use total energy potential surfaces. Non-observables or irrelevant observables ('Mott-Hubbard U') were avoided in the definition of a reasonable delocalisation concept [16]. The concepts used are standard in modern theoretical chemistry but are surprisingly neglected among theoretical solid-state physicists. In fact it is somewhat disturbing that many words and meanings are different in the two sub-fields. Take for example 'Mott-Hubbard U', either defined as I-A or as a Coulomb repulsion. In empty space, the energy of the disproportionation reaction of eq.(1) is equal to $U$, which mathematically is always positive. The disproportionated state is thus predicted as energetically forbidden, in obvious disagreement with experiments. In the condensed phase $U$ cannot be taken from experiments. The Coulomb repulsion is used in a number of other contexts, for example explaining why the Mott insulators are non-conducting. This $\mathrm{U}$ is also positive and large, and this is contradictory to the celebrated 'negative U' scenario for superconductivity. As a result a lot of theoretical effort has gone into explaining reduction of $U$ by some means, for example by electron correlation effects. Cuprates and other high $T_{c}$ systems are therefore said to be 'strongly correlated' in spite of the fact that electron correlation effects are not especially large in these systems.

In superconductivity electron pairs move without activation energy. A ground state must be formed in which pairs of electrons move without resistance. This is only possible if the SDW and CDW are both involved and interact via the vibrational motions. The final ground state vibronic wave function is delocalized and has an energy gap to the first excited state. Other possible ground states are the mixed valent CDW state with quite normal vibrational spectra, or the mono-valent SDW state, also with a normal vibrational spectrum. Softening or disappearance of modes should characterize vibrational spectra of superconductors, as been discussed for cuprates by Prassides [10].

\section{Acknowledgement}

This work was supported by VR, the Swedish Scientific Research Council.

\section{References}

[1] A.W. Sleight, Science 242, 1519 (1988); A.W Sleight in Chemistry of High-Temperature Superconductors, Eds.: D.L. Nelson, M.S. Witting-ham, T.F. George (American Chemical Society), Washington, DC, 1987, pp. 2-12; A.W. Sleight, Acc. Chem. Research 28, 103 (1995).

[2] S. Larsson, Chem. Phys. Lett. 157, 403 (1989).

[3] S. Larsson, Chem. Phys. 236, 133 (1998); S. Larsson, Inorg. Chim. Acta 250, 189 (1996); S. Larsson and A. Klimkans, Int. J. Quant. Chem. 75, 543 (1999); A. Klimkans and S. Larsson, J. Mol. Structure (Theochem.) 527, 203 (2000); S. Larsson, Int. J. Quant. Chem. 90, 1457 (2002).

[4] M.B. Robin and P. Day, Adv. Inorg. Radiochem. 10, 361 (1967).

[5] N.S. Hush, Progr. Inorg. Chem. 8, 391 (1967); G.C. Allen and N.S. Hush, Progr. Inorg. Chem. 8, 357 (1967).

[6] R.A. Marcus, Adv. Phys. Chem. 15, 51 (1964).

[7] T. Holstein, Ann. Phys. 8, 343 (1959).

[8] "Mixed-Valence Compounds; Theory and Applications in Chemistry, Physics, Geology, and Biology" Proceedings of the NATO Advanced Study Institute held at Oxford, England, September 9-21, 1979, Editor David B. Brown (D. Reidel Publishing Company, Dordrecht, Boston, London).

[9] "Mixed Valency Systems: Applications in Chemistry, Physics and Biology" Proceedings of the NATO Advanced Workshop on Mixed Valency Compounds held at Aghia Pelaghia, Crete, Greece, June 10-16, 1990, Editor K. Prassides, NATO ASI Series, vol. 343 (D. Reidel Publishing Company, Dordrecht, Boston, London).

[10] K. Prasssides, page 201 in ref. 8.

[11] L.J. de Jongh, page 223 in ref. 8.

[12] S.B. Piepho, E.R. Krausz, and P.N. Schatz, J. Am. Chem. Soc. 100, 2996 (1978).

[13] K. Prassides, P.N. Schatz, K.Y. Wong, and P. Day, J. Phys. Chem. 90, 5588 (1986).

[14] N.S. Hush, Chem. Phys. 10, 361 (1975).

[15] N.S. Hush in ref.7, p.151-188

[16] S. Larsson and A. Klimkans, Molecular Cryst. and Liquid Cryst. 355, 217 (2001); S. Larsson and A. Klimkans, Int. J. Quant. Chem. 80, 713 (2000). 
[17] P.S. Bagus and H.F. Schaefer III, J. Chem. Phys. 56, 224 (1972).

[18] C. Creutz, Progr. Inorg. Chem. 30, 1 (1983); and further references therein.

[19] J. Bardeen, L.N. Cooper, and J.R. Schrieffer, Phys. Rev. 108, 1175 (1957).

[20] S. Souma, Y. Machida, T. Sato, T. Takahashi, H. Matsui, S.C. Wang, H. Ding, A. Kaminski, J.C. Campuzano, S. Sasaki, and K. Kadowaki, Nature 423, 65 (2003).

[21] A.W. Sleight, J.L. Gillson, and P.E. Bierstedt, Solid State Commun. 17, 27 (1975)

[22] R.J. Cava, B. Batlogg, J.J. Krajewski, R. Farrow, L.W. Rupp, A.E. White, K. Short, W.F. Peck, and T. Kometani, Nature 332, 814 (1988).

[23] D.E. Cox and A.W. Sleight, Solid State Commun. 19, 969 (1976); B. Batlogg, R.J. Cava, L.F. Schneemeyer, and G.P. Espinosa, IBM Jour. Res. Develop. 33, 208 (1989).

[24] K. Prassides and P. Day, J. Chem. Soc. Faraday Trans. 2, 80, 85 (1984).

[25] J.G. Bednorz and K.A. Müller, Z. Phys B 64, 189 (1986).

[26] C.W. Chu, P.H. Hor, R.L. Meng, L. Gao, Z.J. Huang, and Y.Q. Wang, Phys. Rev. Lett. 58, 405 (1987); M.K. Wu, J.R. Ashburn, P.H. Torng, P.H. Hor, R.L. Meng, L. Gao, Z.J. Huang, Y.Q. Wang, and C.W. Chu, Phys. Rev. Lett. 58, 908 (1987).

[27] W. Grochala and R. Hoffmann, Angew. Chem. 113, 2816 (2001); Angew. Chem. Int. Ed. 40, 2742 (2001).

[28] C. Shen, B. Zemva, G.M. Lucier, O. Graudejus, J.A. Allman, and N. Bartlett, Inorg. Chem. 38, 4570 (1999).

[29] N. Elliot and L. Pauling, J. Am. Chem. Soc. 60, 1846 (1938); J.C.M. Tindemans-v. Eijndhoven and G.C. Verschoor, Mater. Res. Bull. 9, 1667 (1974); A.F. Wells, Structural Inorganic Chemistry, third edition, page 374 (Clarendon Press, Oxford 1962).

[30] R. Keller, J. Fenner, and W.B. Holtzapfel, Mater. Res. Bull. 9, 1363 (1974)

[31] P. Day, C. Vettier, and G. Parisot, Inorg. Chem. 17, 2319 (1978).
[32] W. Denner, H. Schulz, and H. D'Amour, Acta Cryst. A35, 360 (1979).

[33] N. Kojima, H. Kitagawa, T. Ban, F. Amita and M. Nakahara, Solid State Commun. 73, 743 (1990); H. Kitagawa, H. Sato, N. Kojima, T. Kikegawa, and O. Shimomura, Solid State Commun. 78, 989 (1991); N. Kojima and H. Kitagawa, J. Chem. Soc. Dalton Trans. 1994, 327-331.

[34] W.L. Yang, V. Brouet, X.J. Zhou, H.J. Choi, S.G. Louie, M.L. Cohen, S.A. Kellar, P.V. Bogdanov, A. Lanzara, A. Goldoni, F. Parmigiani, Z. Hussain, and Z.-X. Shen, Science 300, 303 (2003).

[35] A.F. Hebard, M.J. Rosseinsky, R.C. Haddon, D.W. Murphy, S.H. Glarum, T.T.M. Palstra, A.P. Ramirez, and A.R. Kortan, Nature 350, 600 (1991).

[36] D.W. Murphy, M.J. Rosseinsky, R.M. Fleming, R. Tycko, A.P. Ramirez, R.C. Haddon, T. Siegrist, G. Dabbagh, J.C. Tully, and R.E. Wahlstedt, J. Phys. Chem. Solids 53, 1321 (1992).

[37] J.E. Han and O. Gunnarsson, Physica B 292, 196 (2000).

[38] N.H. Mott, Metal-Insulator Transitions (Taylor and Francis, 1990).

[39] G. Duskesas and S. Larsson, Theoret. Chim. Acta 97, 110 (1997).

[40] S. Larsson in Chemical Physics of Intercalation II, (Plenum Press, New York and London, 1993), Eds. P. Bernier, J.E. Fischer, S. Roth, and S.A. Solin, NATO ASI Series; Series B: Physics, Vol. 305, p. 227-231; S. Larsson and L. RodríguezMonge, Int. J. Quant. Chem. Symp. 27, 655 (1993).

[41] A.P. Ramirez, Supercond. Rev. 1, 1 (1994); M.P. Gelfand, Supercond. Rev. 1, 103 (1994), and further references therein.

[42] V. Brouet, H. Alloul, S. Garaj, and L. Forrö, Phys. Rev. B 66, 155122 (2002).

[43] S. Margadonna and K. Prassides, J. Solid St. Chem. 168, 639 (2002).

[44] Y. Kubozono, Y. Takabayashi, S. Fujiki, S. Kashino, T. Kambe, Y. Iwasa, and S. Emura, Phys. Rev. B 59, 15062 (1999). 